\title{
AN ELEMENTARY WAY TO INTRODUCE A PERRON-LIKE INTEGRAL
}

\author{
Hana Bendová and Jan Malý \\ Charles University, Department of Mathematical Analysis \\ Sokolovská 83, 18600 Prague 8, Czech Republic; haaanja@gmail.com \\ Charles University, Department of Mathematical Analysis \\ Sokolovská 83, 18600 Prague 8, Czech Republic, and \\ J. E. Purkyně University, Department of Mathematics \\ České mládeže 8, 40096 Ústí nad Labem, Czech Republic; maly@karlin.mff.cuni.cz
}

\begin{abstract}
We give an alternative definition of integral at the generality of the Perron integral and propose an exposition of the foundations of integral theory starting from this new definition. Both definition and proofs needed for the development are unexpectedly simple. We show how to adapt the definition to cover the multidimensional and Stieltjes case and prove that our integral is equivalent to the Henstock-Kurzweil(-Stieltjes) integral.
\end{abstract}

\section{Introduction}

The aim of this paper is to built self-contained foundations of the theory of nonabsolutely convergent integral based on a new definition. Our definition is a slight modification of definitions used previously, but provides a possibility of a surprisingly comprehensible development of the theory.

We are focused on integrals which include the Lebesgue integral and integrate all derivatives. The first such construction was done by Denjoy [7] in 1912, shortly followed by Luzin [22]. The integral of Perron [25] from 1914 uses families of major and minor functions instead of a single antiderivative. A "weighted" analogue of the Perron integral is the Perron-Stieltjes integral introduced by Ward [32]. In 1957, Kurzweil [18] introduced a gauge generalized Riemann type integral, which is equivalent to the Perron integral. The same construction was found independently by Henstock [10], see also [11], [14]. The advantage of this construction is that it is based on Riemann sums which are commonly used to illustrate and motivate the concept of integral. Successful attempts have been made to build an elementary course of integration on basis of the Henstock-Kurzweil integral, e.g., [20]. Another curiously simple definition of integral in Perron-like generality is due to Tolstov [30].

The definition of Henstock and Kurzweil is constructive, the integral is constructed as a limit of sums. The idea of descriptive solutions is to impose conditions on a function $F$ to guarantee that it will behave as a kind of indefinite integral of $f$. If we want to include the Lebesgue integral, the condition $F^{\prime}=f$ has to be demanded only almost everywhere. To keep the uniqueness, some additional conditions in spirit

doi:10.5186/aasfm.2011.3609

2010 Mathematics Subject Classification: Primary 26A39; Secondary 26A24, 26A36.

Key words: Perron integral, Henstock-Kurzweil integral.

The second author was supported in part he research project MSM 0021620839 of the Czech Ministry MŠMT and in part by the grant GA ČR 201/09/0067. 
of absolute continuity are usually required. This idea goes back to Luzin, see also [20], [3].

We present a definition of integral which is also equivalent with Perron's definition. Our definition is descriptive, but avoids conditions of absolute continuity type. In addition, no apriori knowledge of null sets is needed. An intermediate step between our integral and the Perron integral is the variational integral. This has been introduced by Henstock [9] and admits various formulations, see, e.g., [12]. In [8], Definition 11.7, we may find a version which can be stated as follows:

A function $f:[a, b] \rightarrow \mathbf{R}$ is variational integrable if there exists a function $F:[a, b] \rightarrow \mathbf{R}$ with the following property: For each $\varepsilon>0$ there exists an increasing function $\varphi_{\varepsilon}:[a, b] \rightarrow \mathbf{R}$ and a strictly positive function $\delta:[a, b] \rightarrow \mathbf{R}$ such that $\left[\varphi_{\varepsilon}\right]_{a}^{b}<\varepsilon$ and for each $x, y \in[a, b]$ we have

$$
|y-x| \leq \delta(x) \Longrightarrow|F(y)-F(x)-f(x)(y-x)| \leq\left|\varphi_{\varepsilon}(y)-\varphi_{\varepsilon}(x)\right| .
$$

The integral of $f$ over $[a, b]$ is then $[F]_{a}^{b}$.

We simplify further the definition of variational integral: we use a single control function and replace the explicit description of $\varepsilon-\delta$ dependence by an ordinary limit. This enables, among others, to use a language in which the definition looks almost like the ordinary definition of (anti)derivative and proofs of tools like integration by parts and change of variables are short and elegant. For experienced mathematicians it is easy to observe that our $M C$-definition is equivalent with the above version of the variational integral. However, we believe that our definition is a significant step to comprehensibility for beginners and students. Moreover, concerning integral calculus based on the variational definition, we have not found any elementary development in literature.

The idea of a single control function appears in analysis also in other contexts: Cornea [5], [6] (see also [21]) uses a control function to modify Perron's construction of solution of the Dirichlet problem in potential theory. Notice that there is a parallel with our construction; also in this case, the original Perron's idea is based on upper and lower functions. Another related concept is that of delta-convex mappings. Originally, (scalar) delta-convex functions are differences of convex functions. Veselý and Zajíček [31] use control functions to generalize delta-convexity to the vector valued case.

Our definition is the following:

Definition 1. Let $I=(a, b) \subset \mathbf{R}$ be an interval and $f, F: I \rightarrow \mathbf{R}$ be functions. We say that $f$ is an $M C$-derivative (monotonically controlled derivative) of $F$ if there exists a strictly increasing function $\varphi: I \rightarrow \mathbf{R}$ (the so-called control function to the pair $(F, f))$ such that

$$
\lim _{y \rightarrow x} \frac{F(y)-F(x)-f(x)(y-x)}{\varphi(y)-\varphi(x)}=0, \quad x \in I .
$$

We also say that $F$ is an $M C$-antiderivative (or an indefinite $M C$-integral) of $f$.

Recall that the $M C$-integral coincides with the Perron integral for which the development of calculus is well known. Despite of this, we hope that it is valuable to present an independent development of the theory. We believe that the concept of $M C$-integral is comprehensible for students-beginners, therefore we want to indicate how the theory of integral can be developed from scratch. However, as we address 
this exposition to experienced mathematicians, our text is not in the style of a course for beginners; such arrangement will be a task for a textbook project.

Any reasonable notion of indefinite integral must have the property that two indefinite integrals of the same function can differ only by an additive constant. This is mostly established by the observation that if $F^{\prime}$ is positive, then $F$ is increasing. This property is valid also for $M C$-differentiation as shown in Section 2 .

Section 3 includes basic tools for integration like integration by parts and change of variables. The results are well known in this generality, see e.g. [26], [19]. However, our definition leads to proofs which are very simple.

In Section 4, we prove the monotone convergence theorem. This opens a gateway for a development of the theory of integral in spirit of Lebesgue integration courses.

The construction of the $M C$-integral can be easily adapted to more general situations. In Section 5, we show a simultaneous generalization to functions of several variables and to Stieltjes integration (in another terminology, integration with respect to Radon measures). The main result of this section is that, even in this generality, the $M C$-integral coincides with a corresponding integral defined by the Henstock-Kurzweil construction (and thus also with the Perron integral). This result is not so hard once we know that the Henstock-Kurzweil integral coincides with the variational integral [11]; the crucial step of this equivalence is the Henstock lemma. In the multidimensional case we observe a remarkable diversity of (nonequivalent) definitions of nonabsolutely convergent integrals and many of them can be rewritten in an $M C$-version. We demonstrate this correspondence on a model case, but the argument can be applied in other situations as well. For various definitions of multidimensional integrals, discussion of problems and further bibliography we refer e.g. to [2], [11], [14], [15], [16], [17], [19], [23], [24], [27], [28].

In contrast with our treatment of the one-dimensional case, we will not concentrate on the development of foundations of the multidimensional theory, because the advantage of simplicity is not so significant here. However, it is worth to mention that once started the development of integration theory with the $M C$-integral, it is possible to proceed to multidimensional integration and obtain the same results as in the theory of multidimensional Henstock-Kurzweil(-Stieltjes) integral. From the didactical point of view, we recommend to restrict attention to the class of absolutely integrable functions, which are exactly the Lebesgue(-Stieljes) integrable functions.

In this paper, we use the term positive for $\geq 0$ whereas $>0$ is called strictly positive. Similar convention applies to the terms increasing and strictly increasing.

\section{2. $M C$-derivatives}

In this section we prove some basic properties of $M C$-differentiation. First, we note that pairs $(F, f)$ such that $f$ is an $M C$-derivative of $F$ form a vector space. It is also evident that any ordinary derivative is an $M C$-derivative. However, there is one serious difference. Ordinary derivatives are unique. If we want to have a concept of derivative general enough to differentiate any indefinite Lebesgue integral, we necessarily lose uniqueness, namely, the derivative is pointwise determined only up to a set of measure zero. At this stage of exposition, we do not need to speak of sets of measure zero; however, it may be useful to note that the exceptional sets are small. To illustrate this phenomenon, we assume that $f$ and $g$ are $M C$-derivatives of $F$, with control functions $\varphi$ and $\psi$, respectively. It is easy to observe then that 
the monotone function $\eta=\varphi+\psi$ has infinite derivative at each point of the set $\{x: f(x) \neq g(x)\}$.

It is useful to notice that if we add an increasing function to a control function to $(F, f)$, we obtain also a control function to $(F, f)$.

If $\varphi$ is a control function to $(F, f)$, then any function of the form $\alpha \varphi+\beta$, where $\alpha, \beta$ are constants, $\alpha>0$, is also a control function to $(F, f)$. Such a modification of a control function is called rescaling.

Proposition 1. Let $F$ be an indefinite $M C$-integral of a function $f$ on an interval $I \subset \mathbf{R}$. Then $F$ is continuous.

Proof. Let $x \in I$. Since $\varphi$ is locally bounded, from (1) we obtain

$$
\lim _{y \rightarrow x}(F(y)-F(x)-f(x)(y-x))=0 .
$$

It follows that $F$ is continuous at $x$.

In the following theorem we prove that an indefinite $M C$-integral of a positive function is increasing.

Theorem 1. Let $F$ be an indefinite $M C$-integral of a function $f \geq 0$ on an open interval $I=\left(a_{0}, b_{0}\right) \subset \mathbf{R}$. Then $F$ is increasing.

Proof. Suppose that there exist $a, b \in I$ such that $a<b$ and $F(b)<F(a)$. By rescaling we find a control function $\varphi$ with $(F+\varphi)(a)>(F+\varphi)(b)$. Let us denote $G=$ $F+\varphi$. Let $a_{1}=a, b_{1}=b$ and $c_{1}=\frac{1}{2}\left(a_{1}+b_{1}\right)$. We choose $\left[a_{2}, b_{2}\right]$ among the intervals $\left[a_{1}, c_{1}\right],\left[c_{1}, b_{1}\right]$ such that $G\left(a_{2}\right)>G\left(b_{2}\right)$. We continue recursively and construct a nested sequence of closed intervals $\left[a_{k}, b_{k}\right]$ such that $b_{k}-a_{k}=2^{1-k}(b-a)$ and $G\left(a_{k}\right)>G\left(b_{k}\right)$. There exists a point $x$ in the intersection of all intervals $\left[a_{k}, b_{k}\right]$. For each $k$ we can choose $x_{k}$ among the points $a_{k}, b_{k}$ such that $x_{k} \neq x$ and $G\left(x_{k}\right)-G(x)$ have the opposite sign to $x_{k}-x$. Since

$$
\lim _{y \rightarrow x} \frac{F(y)-F(x)-f(x)(y-x)}{\varphi(y)-\varphi(x)}=0,
$$

we obtain

$$
\lim _{k \rightarrow \infty} \frac{G\left(x_{k}\right)-G(x)-f(x)\left(x_{k}-x\right)}{\varphi\left(x_{k}\right)-\varphi(x)}=1
$$

however,

$$
\frac{G\left(x_{k}\right)-G(x)}{\varphi\left(x_{k}\right)-\varphi(x)}<0 \quad \text { and } \quad \frac{f(x)\left(x_{k}-x\right)}{\varphi\left(x_{k}\right)-\varphi(x)} \geq 0, \quad k=1,2, \ldots
$$

This is a contradiction.

Corollary 1. If $F, G$ are indefinite $M C$-integrals of a function $f$, then $F-G$ is constant.

Proof. The function $F-G$ is an indefinite $M C$-integral of the zero function and thus it is both increasing and decreasing.

\section{Calculus of $M C$-integral}

Definition 2. If $f$ has an indefinite $M C$-integral $F$ on $(a, b)$ and $F$ has one-sided proper limits $F\left(a_{+}\right), F\left(b_{-}\right)$at the endpoints, then the (definite) $M C$-integral of $f$ 
over $(a, b)$ is defined as the increment of $F$ :

$$
\int_{a}^{b} f(x) d x=[F]_{a}^{b}
$$

where $[F]_{a}^{b}$ denotes $F\left(b_{-}\right)-F\left(a_{+}\right)$. By Corollary 1, this definition is correct, namely, it does not depend on the choice of the indefinite integral. It is obvious that integral is a linear functional. One could also define extended-real-valued integrals this way, but our convention will be that all integrals are real. In this text, all integral symbols refer to $M C$-integration unless specified otherwise.

Proposition 2. Suppose that $f$ is an $M C$-derivative of $F$ and $g$ is an $M C$ derivative of $G$ on $I=(a, b)$. Then $f G+F g$ is an $M C$-derivative of $F G$. Hence the formula on integration by parts

$$
\int_{a}^{b} f(x) G(x) d x=[F G]_{a}^{b}-\int_{a}^{b} F(x) g(x) d x
$$

holds if the increment and the integral on the right are well defined.

Proof. Let $\varphi$ control the pair $(F, f)$ and $\psi$ control the pair $(G, g)$. We have

$$
\begin{aligned}
& F(y) G(y)-F(x) G(x)-(f(x) G(x)+F(x) g(x))(x-a) \\
(2) \quad= & F(y)(G(y)-G(x)-g(x)(y-x))+G(x)(F(y)-F(x)-f(x)(y-x)) \\
& +g(x)(y-x)(F(y)-F(x)-f(x)(y-x))+f(x) g(x)(y-x)^{2}, \quad x, y \in I .
\end{aligned}
$$

By Proposition $1, F$ is continuous. From (2) we see that the pair $(F G, f G+F g)$ is controlled by $\eta(x)=\varphi(x)+\psi(x)+x$. By the assumptions, there exists an indefinite $M C$-integral $H$ of $F g$ with a well defined increment. It follows that $F G-H$ is an indefinite $M C$-integral of $f G$ with a well defined increment.

Proposition 3. Suppose that $F$ is a strictly increasing function which maps open interval $(a, b)$ onto an open interval $(c, d)$. Let $G:(c, d) \rightarrow \mathbf{R}$ be a function. Let $f$ be an $M C$-derivative of $F$ on $(a, b)$ and $g$ be an $M C$-derivative of $G$ on $(c, d)$. Then $x \mapsto g(F(x)) f(x)$ is an $M C$-derivative of $G \circ F$ on $(a, b)$. Hence, the formula on change of variables

$$
\int_{c}^{d} g(y) d y=\int_{a}^{b} g(F(x)) f(x) d x
$$

holds provided that the above assumptions are satisfied and at least one of the integrals converges.

Proof. Let $\varphi$ control the pair $(F, f)$ and $\psi$ control the pair $(G, g)$. We have

$$
\begin{aligned}
& G\left(F\left(x^{\prime}\right)\right)-G(F(x))-g(F(x)) f(x)\left(x^{\prime}-x\right) \\
& =G\left(F\left(x^{\prime}\right)\right)-G(F(x))-g(F(x))\left(F\left(x^{\prime}\right)-F(x)\right) \\
& \left.\quad+g(F(x))\left(F\left(x^{\prime}\right)\right)-F(x)-f(x)\left(x^{\prime}-x\right)\right), \quad x, x^{\prime} \in(a, b) .
\end{aligned}
$$


Since

$$
\begin{aligned}
& \lim _{x^{\prime} \rightarrow x} \frac{G\left(F\left(x^{\prime}\right)\right)-G(F(x))-g(F(x))\left(F\left(x^{\prime}\right)-F(x)\right)}{\psi\left(F\left(x^{\prime}\right)\right)-\psi(F(x))} \\
& =\lim _{y^{\prime} \rightarrow y} \frac{G\left(y^{\prime}\right)-G(y)-g(y)\left(y^{\prime}-y\right)}{\psi\left(y^{\prime}\right)-\psi(y)}=0, \quad x \in(a, b), y=F(x),
\end{aligned}
$$

we easily infer that $\psi \circ F+\varphi$ controls the pair $(G \circ F,(g \circ F) f)$. The statement concerning integration follows immediately.

Lemma 1. Let $F, f:(a, b) \rightarrow \mathbf{R}$ be functions and $\left(a_{k}\right)_{k},\left(b_{k}\right)_{k}$ be sequences of real numbers. Suppose that $a_{k} \searrow a$ and $b_{k} \nearrow b$. If $f$ is an $M C$-derivative of $F$ on each $\left(a_{k}, b_{k}\right)$, then $f$ is an $M C$-derivative on $F$ on $(a, b)$. Moreover, the control function on $(a, b)$ can be chosen to be bounded.

Proof. We may assume that $\left(a_{k}\right)_{k}$ is strictly decreasing and $\left(b_{k}\right)_{k}$ is strictly increasing. For each $k$, let $\varphi_{k}$ be a control function to $(F, f)$ on $\left(a_{k+1}, b_{k+1}\right)$. Then $\varphi_{k}$ is bounded on $\left(a_{k}, b_{k}\right)$ and by rescaling we may assume that $0<\varphi_{k}<1$ on $\left(a_{k}, b_{k}\right)$. Set

$$
\psi_{k}(x)= \begin{cases}0, & x \leq a_{k} \\ \varphi_{k}(x), & a_{k}<x<b_{k} \\ 1, & x \geq b_{k}\end{cases}
$$

Then the function

$$
\varphi=\sum_{k=1}^{\infty} 2^{-k} \psi_{k}
$$

is obviously a bounded control function to $(F, f)$ on $(a, b)$.

Proposition 4. Let $a, b, c \in \mathbf{R}, a<b<c$. Let $f:(a, c) \rightarrow \mathbf{R}$ be $M C$-integrable on $(a, b)$ and $(b, c)$. Then $f$ is $M C$-integrable on $(a, c)$ and

$$
\int_{a}^{c} f(x) d x=\int_{a}^{b} f(x) d x+\int_{b}^{c} f(x) d x .
$$

Proof. Let $F_{1}$ be an indefinite $M C$-integral of $f$ on $(a, b)$ controlled by $\varphi_{1}$ and $F_{2}$ be an indefinite $M C$-integral of $f$ on $(b, c)$ controlled by $\varphi_{2}$. By Lemma 1 we can assume that $\varphi_{1}, \varphi_{2}$ are bounded. Then the one-sided proper limits $F_{1}\left(b_{-}\right), \varphi_{1}\left(b_{-}\right)$, $F_{2}\left(b_{+}\right)$and $\varphi_{2}\left(b_{+}\right)$exist. By adding suitable constants to $F_{i}$ and rescaling $\varphi_{i}, i=1,2$, we can arrange that $F_{1}\left(b_{-}\right)=F_{2}\left(b_{+}\right)=0$ and $\varphi_{1}\left(b_{-}\right)<0<\varphi_{2}\left(b_{+}\right)$. We set

$$
F(x)=\left\{\begin{array}{ll}
F_{1}(x), & x \in(a, b), \\
0, & x=b, \\
F_{2}(x), & x \in(b, c),
\end{array} \quad \varphi(x)= \begin{cases}\varphi_{1}(x), & x \in(a, b), \\
0, & x=b, \\
\varphi_{2}(x), & x \in(b, c) .\end{cases}\right.
$$

We claim that the function $F$ is an indefinite $M C$-integral of $f$ on $(a, c)$ controlled by $\varphi$. Indeed, (1) holds obviously at each $x \in(a, b) \cup(b, c)$. For $x=b$ we use the jump of $\varphi$ at $b$ to observe that the limit in (1) reduces to

$$
\lim _{y \rightarrow x}(F(y)-F(x)-f(x)(y-x)) .
$$

But the last limit clearly vanishes by the continuity of $F$. 


\section{Monotone convergence theorem}

In this section we establish the monotone convergence theorem for the $M C$ integral. This opens a possibility to continue the exposition with Fatou's lemma, dominated convergence theorem and further consequences. Moreover, this result is a crucial step if we want to show that the $M C$-integral includes the Lebesgue integral. Namely, we first observe that constants are integrable over bounded intervals. Using Proposition 4 we obtain that all step (= piecewise constant) functions are integrable. We can define measurable sets as those sets $M$, for which the characteristic function $\chi_{M}$ has an indefinite $M C$-integral. It is well known that a system of sets which contains all intervals and is closed under monotone unions and intersections contains already all Borel sets, see, e.g., [1, 1.3.9]. Alternatively we can use Dynkin systems, see, e.g., [4, Section 1.6]. This is the step in which the monotone convergence theorem below is needed.

If we define the measure of a measurable set $M$ as the integral of the characteristic function of $M$ (or as $\infty$ if this integral diverges), we observe that the "measure" is complete (all subsets of null sets are measurable) and thus the class of all measurable sets contains all Lebesgue measurable sets. The converse inclusion is also true but it is not so simple to prove. Instead of giving a direct proof we note that the $M C$ integral is equivalent with the Henstock-Kurzweil integral (see Section 5), for which this is well known.

Theorem 2. (Monotone convergence theorem) Let $I=(a, b)$ be an open interval and $\left(f_{k}\right)_{k}$ be a sequence of $M C$-integrable functions on $I, f_{k} \nearrow f$. If

$$
\lim _{k \rightarrow \infty} \int_{a}^{b} f_{k}(x) d x<+\infty
$$

then $f$ is $M C$-integrable over $I$ and

$$
\int_{a}^{b} f(x) d x=\lim _{k \rightarrow \infty} \int_{a}^{b} f_{k}(x) d x .
$$

Proof. By subtracting $f_{1}$ we may achieve that $f_{k} \geq 0, k=1,2, \ldots$ For each $k$, let $F_{k}$ be the indefinite $M C$-integral of $f_{k}$ normalized by $F_{k}\left(a_{+}\right)=0$. Then $\left(F_{k}\right)_{k}$ is an increasing sequence of increasing functions and we can define $F=\lim _{k} F_{k}$. From (3) we infer that $F$ is bounded in $(a, b)$. It is also easy to observe that $F\left(b_{-}\right)=$ $\lim _{k \rightarrow \infty} F_{k}\left(b_{-}\right)$and $F\left(a_{+}\right)=0$. Replacing, if necessary, $\left(F_{k}\right)_{k}$ by a subsequence, we may assume that

$$
F_{k}\left(b_{-}\right)>F\left(b_{-}\right)-2^{-k} .
$$

Since $f_{j}-f_{k} \geq 0$ for $j>k$, the function $F_{j}-F_{k}$ is increasing by Theorem 1 . Passing to the limit we obtain that each $F-F_{k}$ is increasing. For each $k$, let $\varphi_{k}$ be a control function to $\left(F_{k}, f_{k}\right)$. By Lemma 1 we may assume that $\varphi_{k}$ is bounded and thus it may be rescaled to satisfy $0<\varphi_{k}<1$. We set

$$
\varphi(x)=\sum_{k=1}^{\infty} 2^{-k} \varphi_{k}(x)+\sum_{k=1}^{\infty} k\left(F(x)-F_{k}(x)\right)+x .
$$

From (4) we infer that $\varphi$ is finite in $(a, b)$; it is obviously strictly increasing. We claim that $\varphi$ controls $(F, f)$. We choose $x \in I$ and $\varepsilon>0$. We find an integer $k>0$ such 
that $\frac{1}{k}<\varepsilon$ and $f(x)-f_{k}(x)<\varepsilon$. Then we estimate

$$
\begin{aligned}
& F(y)-F(x)-f(x)(y-x)=\left(F_{k}(y)-F_{k}(x)-f_{k}(x)(y-x)\right) \\
& \quad+\left(F(y)-F(x)-\left(F_{k}(y)-F_{k}(x)\right)\right)+\left(f_{k}(x)-f(x)\right)(y-x) \\
& \leq\left(F_{k}(y)-F_{k}(x)-f_{k}(x)(y-x)\right)+\left(\frac{1}{k}+\varepsilon\right)(\varphi(y)-\varphi(x)) .
\end{aligned}
$$

Therefore

$$
\limsup _{y \rightarrow x} \frac{F(y)-F(x)-f(x)(y-x)}{\varphi(y)-\varphi(x)} \leq 2 \varepsilon,
$$

which verifies the claim. Hence $F$ is an indefinite $M C$-integral of $f$ and

$$
\int_{a}^{b} f(x) d x=[F]_{a}^{b}=\lim _{k \rightarrow \infty}\left[F_{k}\right]_{a}^{b}=\lim _{k \rightarrow \infty} \int_{a}^{b} f_{k}(x) d x .
$$

\section{A comparison with the Henstock-Kurzweil integral}

In this section we show that our $M C$-integral coincides with the HenstockKurzweil integral. This will be done in the framework of multidimensional Stieltjes integration.

The multidimensional integration requires the language of interval functions. For an introduction to manipulation with interval functions, in particular to their differentiation, we refer to Saks [29]. By an interval in $\mathbf{R}^{n}$ we mean a Cartesian product of one-dimensional intervals. Let $\mathscr{I}$ denote the collection of all nondegenerate bounded closed intervals in $\mathbf{R}^{n}$. A finite set $\mathscr{D} \subset \mathscr{I}$ is called a partition of an interval $I \in \mathscr{I}$ if the intervals from $\mathscr{D}$ are nonoverlapping (i.e., have disjoint interiors) and $\bigcup_{Q \in \mathscr{D}} Q=I$. A function $\mathbf{F}: \mathscr{I} \rightarrow \mathbf{R}$ is said to be

- additive, if for each interval $I \in \mathscr{I}$ and each partition $\mathscr{D}$ of $I$ we have

$$
\sum_{Q \in \mathscr{D}} \mathbf{F}(Q)=\mathbf{F}(I)
$$

- superadditive, if for each interval $I \in \mathscr{I}$ and each partition $\mathscr{D}$ of $I$ we have

$$
\sum_{Q \in \mathscr{D}} \mathbf{F}(Q) \leq \mathbf{F}(I)
$$

There are many possibilities how to modify the definition below, for example to require some "regularity" of intervals in the limiting process like in [23]. This will yield a variety of non-equivalent integrals. We illustrate our approach on the simplest model case. We consider only indefinite integrals. The definite integrals over unbounded intervals can be defined by an appropriate limit process.

Definition 3. Let $f: \mathbf{R}^{n} \rightarrow \mathbf{R}$ be a function and $\mathbf{F}: \mathscr{I} \rightarrow \mathbf{R}, \mathbf{G}: \mathscr{I} \rightarrow \mathbf{R}$ be additive interval functions. We say that $f$ is an $M C$-derivative of $\mathbf{F}$ with respect to $\mathbf{G}$, or that $\mathbf{F}$ is an indefinite $M C$-integral of $f$ with respect to $\mathbf{G}$, if there exists a superadditive interval function $\boldsymbol{\Phi}>0$ (called a control function) such that for each $x \in \mathbf{R}^{n}$ and for each sequence $\left(Q_{k}\right)_{k}$ of intervals from $\mathscr{I}$ such that $x \in \bigcap_{k} Q_{k}$ and $\operatorname{diam} Q_{k} \rightarrow 0$ we have

$$
\lim _{k \rightarrow \infty} \frac{\mathbf{F}\left(Q_{k}\right)-f(x) \mathbf{G}\left(Q_{k}\right)}{\mathbf{\Phi}\left(Q_{k}\right)}=0
$$


If we require $\boldsymbol{\Phi}$ in the definition of $M C$-derivative ( $M C$-integral) to be additive, we label the result as an $A M C$-derivative (an $A M C$-integral).

Each superadditive interval function $\Phi>0$ satisfies

$$
P, Q \in \mathscr{I}, \quad P \subset Q \Longrightarrow \boldsymbol{\Phi}(P) \leq \boldsymbol{\Phi}(Q) .
$$

Therefore the terminology "monotonically controlled" is reasonable again.

Remark 1. Additive interval functions in $\mathbf{R}$ have the form

$$
\mathbf{G}([a, b])=G(b)-G(a),
$$

where $G: \mathbf{R} \rightarrow \mathbf{R}$ is an "ordinary" function. For the one-dimensional Stieltjes differentiation and integration, (5) reduces to

$$
\lim _{y \rightarrow x} \frac{F(y)-F(x)-f(x)(G(y)-G(x))}{\varphi(y)-\varphi(x)}=0 .
$$

There is no need to use superadditive control functions in $\mathbf{R}$ because each superadditive function $\boldsymbol{\Phi}>0$ is easily majorized by an additive function, using the increasing function

$$
\varphi(x)= \begin{cases}\boldsymbol{\Phi}([0, x]), & x>0, \\ 0, & x=0, \\ -\boldsymbol{\Phi}([x, 0]), & x<0 .\end{cases}
$$

In higher dimension, the relation between the $M C$ and $A M C$ definition is not so clear, see [12]. Since the $M C$-integral includes the $A M C$-integral, we prefer $M C$ integration. On the other hand, the notion of additive functions may seem to be more elementary and for the purpose of absolute integration the concept of $A M C$ integration is sufficient.

We recall the definition of $H K$-integral with respect to an additive interval function $\mathbf{G}$ as defined, e.g., in [19].

Definition 4. Let $I \in \mathscr{I}$ be an interval. A tagged partition of $I$ is defined as a couple $(\mathscr{D}, \tau)$ where $\mathscr{D}$ is a partition of $I$ and $\tau: \mathscr{D} \rightarrow \mathbf{R}^{n}$ is a mapping such that

$$
\tau(Q) \in Q, \quad Q \in \mathscr{D} .
$$

The condition (6) is not always required in literature (it should be dropped for McShane integration), but it should be assumed for the purpose of $H K$-integration. We identify a tagged partition $(\mathscr{D}, \tau)$ with the set $\{(Q, x): Q \in \mathscr{D}, x=\tau(Q)\}$. By a gage we mean a strictly positive function $\delta: \mathbf{R}^{n} \rightarrow \mathbf{R}$. Given a gage $\delta$, we say that a tagged partition $(\mathscr{D}, \tau)$ of $I$ is $\delta$-fine if for each $(Q, x) \in(\mathscr{D}, \tau)$ we have $\operatorname{diam} Q<\delta(x)$. Let $f: I \rightarrow \mathbf{R}$ be a function and $\alpha \in \mathbf{R}$. We say that $\alpha$ is an $H K$-integral (Henstock-Kurzweil version of the Stieltjes integral) of $f$ over $I$ with respect to $\mathbf{G}$ if for each $\varepsilon>0$ there exists a gage $\delta$ such that for each $\delta$-fine partition $(\mathscr{D}, \tau)$ of $I$ we have

$$
\left|\sum_{(Q, x) \in(\mathscr{D}, \tau)} f(x) \mathbf{G}(Q)-\alpha\right|<\varepsilon .
$$

The $H K$-integral is unique if it exists. We define the indefinite HK-integral of $f: \mathbf{R}^{n} \rightarrow \mathbf{R}$ as the interval function which assigns to each $Q \in \mathscr{I}$ the $H K$-integral of $f$ over $Q$ with respect to $\mathbf{G}$. It is an additive interval function. 
Definition 5. Let $\Psi: \mathscr{I} \times \mathbf{R}^{n} \rightarrow \mathbf{R}$ be a function and $\delta$ be a gage. The $\delta$ variation of $\Psi$ is defined as

$$
V_{\delta}(P, \Psi)=\sup \left\{\sum_{(Q, x) \in(\mathscr{D}, \tau)}|\Psi(Q, x)|:(\mathscr{D}, \tau) \text { is a } \delta \text {-fine partition of } P\right\}, \quad P \in \mathscr{I} .
$$

If the interval function $V_{\delta}(\cdot, \Psi)$ is finite, then it is superadditive.

The following statement establishes the equivalence of the Henstock-Kurzweil integral and the so-called variational integral. The only if part is known as Henstock's lemma. For the proof see, e.g., [13], Theorem 44.6, [14].

Proposition 5. Let $\mathbf{F}, \mathbf{G}$ be additive interval functions on $\mathscr{I}, \mathbf{G} \geq 0$. Let $f: \mathbf{R}^{n} \rightarrow \mathbf{R}$ be a function. Let $\Psi: \mathscr{I} \times \mathbf{R}^{n} \rightarrow \mathbf{R}$ is defined by

$$
\Psi(Q, x)=f(x) \mathbf{G}(Q)-\mathbf{F}(Q), \quad Q \in \mathscr{I}, x \in \mathbf{R}^{n} .
$$

Then $\mathbf{F}$ is an indefinite $H K$ integral of $f$ with respect to $\mathbf{G}$ if and only if

$$
\inf \left\{V_{\delta}(I, \Psi): \delta \text { is a gage }\right\}=0
$$

for each $I \in \mathscr{I}$.

Finally, we are ready to compare our $M C$-definition of integral with the $H K$ integral.

Theorem 3. Let $f: \mathbf{R}^{n} \rightarrow \mathbf{R}$ be a function and $\mathbf{F}: \mathscr{I} \rightarrow \mathbf{R}$ be an interval function. Then $\mathbf{F}$ is an indefinite $M C$-integral of $f$ with respect to $\mathbf{G}$ if and only if $\mathbf{F}$ is an indefinite $H K$-integral of $f$ with respect to $\mathbf{G}$.

Proof. Let $\boldsymbol{\Phi}: \mathscr{I} \rightarrow \mathbf{R}$ be a control function to $(\mathbf{F}, f)$ with respect to $\mathbf{G}$. Choose $I \in \mathscr{I}$ and $\varepsilon>0$. For each $x \in I$ there exists $\delta(x)>0$ such that for all $Q \in \mathscr{I}$ containing $x$ with $\operatorname{diam} Q<\delta$ we have

$$
|\mathbf{F}(Q)-f(x) \mathbf{G}(Q)|<\varepsilon \mathbf{\Phi}(Q) .
$$

We claim that $\delta$ is the desired gage. If $(\mathscr{D}, \tau)$ is a $\delta$-fine partition of an interval $I \in \mathscr{I}$, then

$$
\begin{aligned}
\left|\sum_{(Q, x) \in(\mathscr{D}, \tau)} f(x) \mathbf{G}(Q)-\mathbf{F}(I)\right| & =\left|\sum_{(Q, x) \in(\mathscr{D}, \tau)}(f(x) \mathbf{G}(Q)-\mathbf{F}(Q))\right| \\
& \leq \sum_{(Q, x) \in(\mathscr{D}, \tau)}|(f(x) \mathbf{G}(Q)-\mathbf{F}(Q))| \\
& \leq \sum_{(Q, x) \in(\mathscr{D}, \tau)} \varepsilon \mathbf{\Phi}(Q)<\varepsilon \mathbf{\Phi}(I) .
\end{aligned}
$$

It follows that $\mathbf{F}$ is an indefinite $H K$-integral of $f$ with respect to $\mathbf{G}$. Conversely, suppose that $\mathbf{F}$ is an indefinite $H K$-integral of $f$ with respect to $\mathbf{G}$ and denote

$$
\Psi(Q, x)=f(x) \mathbf{G}(Q)-\mathbf{F}(Q), \quad(Q, x) \in \mathscr{I} \times \mathbf{R}^{n} .
$$

Consider the intervals $I_{k}=[-k, k]^{n}$. Using the Henstock lemma (Proposition 5), for each integer $k>0$ we find a gage $\delta_{k}$ on $I_{k}$ such that

$$
V_{\delta_{k}}\left(I_{k}, \Psi\right) \leq 2^{-k}
$$


Set

$$
\boldsymbol{\Phi}(Q)=|Q|+\sum_{k=1}^{\infty} k V_{\delta_{k}}\left(Q \cap I_{k}\right) .
$$

Then $\boldsymbol{\Phi}(Q)$ is a strictly positive finite superadditive function on $\mathscr{I}$. Given $x \in \mathbf{R}$ and $\varepsilon>0$ we find an integer $k>1$ such that $\frac{1}{k}<\varepsilon$ and $x \in I_{k-1}$. Let $Q \in \mathscr{I}$ be such that $x \in Q \subset I_{k}$ and $\operatorname{diam} Q<\delta_{k}(x)$. Then

$$
|f(x) \mathbf{G}(Q)-\mathbf{F}(Q)|=|\Psi(x, Q)| \leq V_{\delta_{k}}(Q, \Psi) \leq \frac{1}{k} \mathbf{\Phi}(Q)<\varepsilon \boldsymbol{\Phi}(Q) .
$$

This shows that $\mathbf{F}$ is an indefinite $M C$-integral of $f$ with respect to $\mathbf{G}$ controlled by $\Phi$.

Acknowledgment. We thank Luděk Zajíček for valuable discussion and comments.

\section{References}

[1] Ash, R. B.: Measure, integration, and functional analysis. - Academic Press, New York, 1972.

[2] Bongionno, B.: The Henstock-Kurzweil integral, - In: Handbook of measure theory I, II, North-Holland, Amsterdam, 2002, 587-615.

[3] Bongionno, B., W. F. Pfeffer, and B. S. Thomson: A full descriptive definition of the gauge integral. - Canad. Math. Bull. 39:4, 1996, 390-401.

[4] Cohn, D. L.: Measure theory. - Birkhäuser, Boston, Mass., 1980.

[5] Cornea, A.: Résolution du problème de Dirichlet et comportement des solutions à la frontière à l'aide des fonctions de contrôle. - C. R. Acad. Sci. Paris Sér. I Math. 320:2, 1995, 159-164.

[6] Cornea, A.: Applications of controlled convergence in analysis. - In: Analysis and topology, World Sci. Publ., River Edge, NJ, 1998, 257-275.

[7] Denjoy, A.: Une extension de l'intégrale de M. Lebesgue. - C. R. Acad. Sci. Paris Sér. I Math. $154,1912,859-862$.

[8] Gordon, R. A.: The integrals of Lebesgue, Denjoy, Perron, and Henstock. - Grad. Stud. Math. 4, Amer. Math. Soc., Providence, RI, 1994.

[9] Henstock, R.: A new descriptive definition of the Ward integral. - J. London Math. Soc. 35, 1960, 43-48.

[10] Henstock, R.: Definitions of Riemann type of the variational integrals. - Proc. London Math. Soc. (3) 11, 1961, 402-418.

[11] Henstock, R.: Theory of integration. - Butterworths, London, 1963.

[12] Henstock, R.: Majorants in variational integration. - Canad. J. Math. 18, 1966, 49-74.

[13] Henstock, R.: Linear analysis. - Plenum Press, New York, 1967.

[14] Henstock, R.: The general theory of integration. - Oxford Math. Monogr., Oxford Sci. Publ., The Clarendon Press, Oxford Univ. Press, New York, 1991.

[15] JARník, J., and J. KuRzweiL: A nonabsolutely convergent integral which admits transformation and can be used for integration on manifolds. - Czechoslovak Math. J. 35(110):1, 1985, $116-139$.

[16] Jarník, J., J. Kurzweil, and Š. Schwabik: On Mawhin's approach to multiple nonabsolutely convergent integral. - Časopis Pěst. Mat. 108:4, 1983, 356-380.

[17] Jurkat, W.B.: The divergence theorem and Perron integration with exceptional sets. Czechoslovak Math. J. 43(118):1, 1993, 27-45. 
[18] KurzweIL, J.: Generalized ordinary differential equations and continuous dependence on a parameter. - Czechoslovak Math. J. 7(82), 1957, 418-449.

[19] KurzweIL, J.: Nichtabsolut konvergente Integrale. - Teubner-Texte zur Mathematik 26, BSB B. G. Teubner Verlagsgesellschaft, Leipzig, 1980.

[20] Lee, P. Y., and R. VÝBornÝ: Integral: an easy approach after Kurzweil and Henstock. Austral. Math. Soc. Lect. Ser. 14, Cambridge Univ. Press, Cambridge, 2000.

[21] Lukeš, J., J. Malý, I. Netuka, and J. SpurnÝ: Integral representation theory. Applications to convexity, Banach spaces and potential theory. - Stud. in Math. 35, Walter de Gruyter, Berlin, New York, 2010.

[22] Lusin, N.: Sur les propriétés de l'intégrale de M. Denjoy. - C. R. Acad. Sci. Paris Sér. I Math. 155, 1912, 1475-1478.

[23] Mawhin, J.: Generalized multiple Perron integrals and the Green-Goursat theorem for differentiable vector fields. - Czechoslovak Math. J. 31(106):4, 1981, 614-632.

[24] Novikov, A., and W. F. Pfeffer: An invariant Riemann type integral defined by figures. Proc. Amer. Math. Soc. 120:3, 1994, 849-853.

[25] Perron, O.: Über den Integralbegriff. - Sitzungsber. Heidelberg Akad. Wiss. A16, 1914, 1-16.

[26] Pfeffer, W.F.: Integration by parts for the generalized Riemann-Stieltjes integral. - J. Austral. Math. Soc. Ser. A 34:2, 1983, 229-233.

[27] Pfeffer, W. F.: The Riemann approach to integration. - Cambridge Tracts in Math. 109, Cambridge Univ. Press, Cambridge, 1993.

[28] Pfeffer, W. F.: Derivation and integration. - Cambridge Tracts in Math. 140, Cambridge Univ. Press, Cambridge, 2001.

[29] SAKs, S.: Theory of the integral. - Dover Publ. Inc., 2nd revised edition, New York, 1964.

[30] Tolstov, G. P.: Parametric differentiation and the narrow Denjoy integral. - Mat. Sb. (N.S.) 53(95), 1961, 387-392.

[31] Veselý, L., and L. ZaJíčEK: Delta-convex mappings between Banach spaces and applications. - Dissertationes Math. (Rozprawy Mat.) 289, 1989, 1-52.

[32] Ward, A. J.: The Perron-Stieltjes integral. - Math. Z. 41, 1936, 578-604.

Received 16 February 2010 